\title{
Generating Sequences of Acoustic Scintillations
}

\author{
F. Rietdijk ${ }^{1,2)}$, J. Forssén ${ }^{2)}$, K. Heutschi ${ }^{1)}$ \\ 1) Laboratory for Acoustics/Noise Control, Swiss Federal Laboratories for Materials Science and Technology, \\ 8600 Duebendorf, Switzerland. frederik.rietdijk@empa.ch \\ 2) Division of Applied Acoustics, Chalmers University of Technology, SE-41296 Gothenburg, Sweden. \\ jens.forssen@chalmers.se
}

\begin{abstract}
Summary
Spatial and temporal inhomogeneities in temperature and wind velocity affect sound propagation resulting in amplitude and phase fluctuations called scintillations. A computationally efficient method is presented to generate sequences of scintillations. The method, already used in the field of wireless communication to predict the performance of wireless communication links, could be used in the field of acoustics to create more perceptually valid auralizations. A Gaussian spectrum and a spherical wavefront is considered, but the method can also be used in combination with other spectra like the Von Karman spectrum as well as plane waves. Two examples are given, one is a pure tone affected by the scintillations and the other is an auralization of an aircraft fly-over. The effect of the transverse speed of the source is demonstrated as well.
\end{abstract}

(C) 2017 The Author(s). Published by S. Hirzel Verlag · EAA. This is an open access article under the terms of the Creative Commons Attribution (CC BY 4.0) license (https://creativecommons.org/licenses/by/4.0/).

PACS no. 43.28.Gq

\section{Introduction}

In an outdoor situation, spatial and temporal variations in temperature and wind velocity cause small changes in the refractive index. As waves pass through the atmosphere, the index-of-refraction variations in effect cause scintillations, i.e. fluctuations in the received intensity of the wave. Scintillations affect both sound and electromagnetic waves. They are a major limitation for astronomical observations using Earth-based telescopes and also reduce performance of wireless communication systems. Scintillations can also be noticed when hearing sound emitted by a source at a large distance, like for example by an aircraft or distant windturbine [1].

Within the SONORUS project [2], a tool was developed to auralize the sound of aircraft flying over urban areas [3]. Scintillations affect the audible sound, and it is presumed that including scintillations increases the perceived realism of auralizations. Earlier work introduced a coherence factor to include the loss of coherence due to phase fluctuations $[4,5,6]$. Fluctuations due to turbulence have also been included in auralizations by simulating the amplitude modulations that were observed in measurements $[1,7]$.

In this paper a method is presented to generate time series of sound pressure fluctuations caused by line-ofsight propagation through a weakly turbulent atmosphere. Novel in this field, the method includes both log-amplitude

Received 12 October 2016,

accepted 2 January 2017. and phase fluctuations. The presented method is based on [8] where it was used to predict the performance of wireless communication links, and [9] where it was used to determine the influence of turbulence on the performance of a barrier. The method was used by the authors to increase the perceptual validity of auralizations $[10,11]$.

\section{Wave propagation in random media}

\subsection{Rytov approximation}

Variations in temperature and wind in both position $\mathbf{r}$ and time $t$ cause variations in the refractive index field $n(\mathbf{r}, t)$. We are interested in how these variations affect wave propagation and follow [12] and [8], but instead of electromagnetic waves, we consider sound waves. We consider for now spatial variations only, and as a starting point we use the Helmholtz equation

$$
\left(\nabla^{2}+k^{2} n^{2}(\mathbf{r})\right) p(\mathbf{r})=0,
$$

with pressure $p$, wavenumber $k$, and refractive-index field

$$
n(\mathbf{r})=n_{0}+n_{1}(\mathbf{r})
$$

with mean value $n_{0}=E[n(\mathbf{r})]=1$ and first-order perturbation $n_{1}(\mathbf{r}) \ll 1$. Merging equations (1) and (2) results in

$$
\left(\nabla^{2}+k^{2}\left(n_{0}+n_{1}(\mathbf{r})\right)^{2}\right) p(\mathbf{r})=0
$$


For weak fluctuations, an approximation to equation (3) for small $n_{1}$ is used. The Rytov solution to equation (3) is

$$
p=\exp \left(\psi_{0}+\psi_{1}+\psi_{2}+\ldots\right)=\exp (\psi),
$$

where $\psi_{0}$ is the complex phase of the unperturbed wave in free space, and $\psi_{1}$ and $\psi_{2}$ respectively first-order and second-order complex phase perturbations.

We are interested in the effect of first-order perturbations $n_{1}$, on the sound pressure, and therefore write $\psi=$ $\psi_{0}+\psi_{1}$. The refractive index $n$ is written in terms of an average $\langle n\rangle$ and fluctuation $n_{1}$, with

$$
\delta n=\left(1+n_{1}\right)^{2}-1=2 n_{1}+n_{1}^{2} .
$$

As derived in [12], $\psi_{1}$ satisfies the following integral equation

$$
\begin{aligned}
\psi_{1}(\mathbf{r})=\frac{1}{p_{0}(\mathbf{r})} \int_{V^{\prime}} G\left(\mathbf{r}-\mathbf{r}^{\prime}\right) \\
\cdot\left[\nabla \psi_{1} \cdot \nabla \psi_{1}+k^{2} \delta n\right] p_{0}\left(\mathbf{r}^{\prime}\right) \mathrm{d} V^{\prime},
\end{aligned}
$$

where $G\left(\mathbf{r}-\mathbf{r}^{\prime}\right)$ is the free field Green's function. By iteration a series solution can be obtained. For the first iteration we set $\psi_{1}=0$ inside the integral and obtain the first Rytov solution

$$
\psi_{10}(\mathbf{r})=\frac{k^{2}}{p_{0}(\mathbf{r})} \int_{V^{\prime}} G\left(\mathbf{r}-\mathbf{r}^{\prime}\right) \delta n\left(\mathbf{r}^{\prime}\right) p_{0}\left(\mathbf{r}^{\prime}\right) \mathrm{d} V^{\prime},
$$

where $p_{0}(\mathbf{r})$ is the unperturbed sound pressure field. The sound pressure after the first iteration is then

$$
p(\mathbf{r})=\mathrm{e}^{\left(\psi_{0}+\psi_{10}\right)}=p_{0}(\mathbf{r}) \mathrm{e}^{\left(\psi_{10}\right)} .
$$

The first-order complex phase perturbation $\psi_{1}$ can be understood as a sum of waves, generated at various points $\mathbf{r}^{\prime}$ throughout the scattering volume $V^{\prime}$. The strength of each of these waves is proportional to the product of the unperturbed field term $p_{0}$, and the refractive-index perturbation $\delta n$ at a point $\mathbf{r}^{\prime}[8]$.

\subsection{Amplitude and phase fluctuations}

We now want to find expressions for the log-amplitude and phase fluctuations, and will use Rytov's first solution. We approximate the refractive-index fluctuation as

$$
\delta n=2 n_{1}+n_{1}^{2} \simeq 2 n_{1}
$$

and write

$$
p(\mathbf{r})=A(\mathbf{r}) \mathrm{e}^{\mathrm{j} S(\mathbf{r})}, \quad p_{0}(\mathbf{r})=A_{0}(\mathbf{r}) \mathrm{e}^{\mathrm{j} S_{0}(\mathbf{r})},
$$

where $A$ and $S$ are respectively the amplitude and phase of the fluctuating field $p(\mathbf{r})$, and obtain for the first order perturbations

$$
\psi_{1}(\mathbf{r})=\chi+\mathrm{j} S=\log \left(A / A_{0}\right)+\mathrm{j}\left(S-S_{0}\right) .
$$

In this expression $\chi$ and $S$ represent respectively the logamplitude fluctuation and phase fluctuation.

By applying the central limit theorem to the first Rytov solution, it follows that the complex phase follows a normal probability distribution [8]. This is an important result to keep in mind when generating sequences of fluctuations.

\subsection{Amplitude and phase covariance}

The log-amplitude and phase fluctuations are considered to be the result of a random temperature fluctuation field. A characteristic of a random function or field is its correlation function [13]. The spatial correlation function of a random field $f(\mathbf{r})$, as function of distance $\mathbf{r}=\mathbf{r}_{2}-\mathbf{r}_{1}$ between observation points $\mathbf{r}_{1}$ and $\mathbf{r}_{2}$, is defined as

$$
C\left(\mathbf{r}_{1}, \mathbf{r}_{2}\right)=\left\langle f\left(\mathbf{r}_{1}\right) f\left(\mathbf{r}_{2}\right)\right\rangle .
$$

In a homogeneous and isotropic random field the correlation function $C(r)$ depends only on the distance $r=$ $\left\|\mathbf{r}_{2}-\mathbf{r}_{1}\right\|$ between the observation points and not the path $\mathbf{r}=\mathbf{r}_{2}-\mathbf{r}_{1}$ [14]. Note that at this point, the atmosphere is assumed frozen in time, i.e., variations are only spatially, not temporal.

We would like to obtain expressions for the covariance functions of the log-amplitude and phase fluctuations. A specific part of the turbulence spectrum can be approximated with a Gaussian correlation function

$$
C_{\mu}=\left\langle\mu\left(r_{1}\right) \mu\left(r_{2}\right)\right\rangle=\sigma_{\mu}^{2} \mathrm{e}^{-x^{2} / L^{2}}
$$

where $\sigma_{\mu}^{2}$ is the variance of the dynamic refractive index, $x=r_{1}-r_{2}$ the distance between two points in space and $L$ the correlation distance or length [12].

We shall now consider a line-of-sight situation where $d$ is the distance between the source and a receiver pair along the wave propagation direction, and $\rho$ the spatial separation of the receivers transverse to the wave propagation direction.

If the correlation length $L$ is much smaller than the Fresnel zone size of the sound $\sqrt{\lambda d}$, then the log-amplitude and phase variance scale with $\sigma_{\chi}^{2}=\sigma_{S}^{2} \sim k^{2} d$ [12] and the variances of the fluctuations are given by [15]

$$
\sigma_{\chi}^{2}=\sigma_{S}^{2}=\frac{\sqrt{\pi}}{2} \sigma_{\mu}^{2} k^{2} d L
$$

For spherical waves the covariances of the fluctuations, $B_{\chi}(\rho)$ and $B_{S}(\rho)$, normalized to their variances, are given by

$$
\frac{B_{\chi}(\rho)}{\sigma_{\chi}^{2}}=\frac{B_{S}(\rho)}{\sigma_{S}^{2}}=\frac{\Phi(\rho / L)}{\rho / L}=C_{s p}(\rho),
$$

where

$$
\begin{aligned}
\Phi(\rho / L) & =\int_{0}^{\rho / L} \exp \left(-u^{2}\right) \mathrm{d} u \\
& =\frac{1}{2} \sqrt{\pi} \operatorname{erf}(\rho / L),
\end{aligned}
$$

and erf is the error function. The covariance functions of the fluctuations $B_{\chi}(\rho)$ and $B_{S}(\rho)$ are thus

$$
B_{\chi}(\rho)=B_{S}(\rho)=\frac{\sqrt{\pi}}{2} \sigma_{\mu}^{2} k^{2} d L \frac{\Phi(\rho / L)}{\rho / L} .
$$


Still assuming Taylor's hypothesis regarding frozen turbulence, we can perform a space-to-time conversion of the correlation and covariance functions to obtain $C(\tau)$ and $B(\tau)$ respectively. The turbulence correlation time is given by

$$
\tau_{0}=\frac{L}{v_{\perp}}
$$

and the transverse time lag by

$$
\tau=\frac{\rho}{v_{\perp}}
$$

where $v_{\perp}$ is the transverse speed, corresponding to e.g. the mean speed at which the field is carried by the wind transverse to the wave propagation direction.

In this paper we will continue to use the covariance for spherical waves and a Gaussian spectrum, because the Gaussian spectrum is the simplest model to work with and computationally least demanding, but the method can also be used with covariance functions that describe other turbulence spectra, like e.g. the Von Karman spectrum [16].

\subsection{Propagation in the turbulent atmosphere as a multichannel}

The fluctuations in the atmosphere are temporal and/or spatial. Therefore, to model sound propagation of a signal $x(t)$ through such an atmosphere a time-varying channel is necessary. The received signal $y(t)$ consists of a line-ofsight contribution and additional contributions from scattering, together forming a multichannel [8]. Ignoring beam spreading, we can write this as

$$
y(t)=\sum_{n} \alpha_{s c_{n}}(t) x\left(t-\tau_{n}(t)\right)
$$

where $\alpha_{s c_{n}}(t)$ is the time-varying scintillation sequence representing the effect of the pressure fluctuations on the $n$ th-multipath component, and $\tau_{n}$ the propagation delay of the $n$th component relative to the propagation delay in an undisturbed atmosphere. Assuming the spread in propagation delay over the channels is small compared to the inverse of the signal bandwidth, so that $x\left(t-\tau_{n}\right) \approx$ $x(t-\tau(t))$, then

$$
y(t)=x(t-\tau(t)) \sum_{n} \alpha_{s c_{n}}(t)
$$

Because the attenuation is very similar for the different multipaths, we can write that

$$
y(t)=x(t-\tau(t)) \alpha_{s c}(t) .
$$

Therefore, the received signal is obtained by shifting the emitted signal $x(t)$ with a time-varying propagation delay $\tau(t)$, and multiplying the result with a time-varying gain $\alpha_{s c}(t)$.

\section{Generating sequences of scintillations}

\subsection{Design of scintillation sequence}

We will now design a sequence of scintillations and for that we need to know the statistical distribution and power spectral density $\left|H_{B}(f)\right|^{2}$ of the desired sequence. As mentioned before the fluctuations are Gaussian-distributed. We can therefore generate a sequence with the correct distribution and power spectral density by convolving a random unit variance Gaussian signal $z(t)$ with the impulse response $h_{B}(t)$ of the designed filter.

$$
\chi(t)=S(t)=\left(h_{B} * z\right)(t)
$$

The power spectral density $\left|H_{B}(f)\right|^{2}$ of a random sequence forms a Fourier pair with its autocorrelation function $R(\tau)$ through the Wiener-Khinchin theorem ${ }^{1}$. Assuming $R(\tau) \cong B_{\chi}(\tau)=B_{S}(\tau)$, the power spectral density of $\chi$ and $S$ is

$$
\left|H_{B}(f)\right|^{2}=\int_{-\infty}^{\infty} B(\tau) \exp (-\mathrm{j} \omega \tau) \mathrm{d} \tau .
$$

This filter has zero phase and is non-causal. To create a causal filter with constant group delay $\alpha$ we can shift the peak in the impulse response by adding a linear-phase factor corresponding to 90 degrees

$$
H_{B}(f)=\left|H_{B}(f)\right| \cdot \exp (-j 2 \pi f \alpha) .
$$

The impulse response of the filter is finally obtained through the Inverse Fourier Transform

$$
h_{B}(t)=\int_{-\infty}^{\infty} H_{B}(f) \exp (+\mathrm{j} \omega \tau) \mathrm{d} \tau
$$

\subsection{Discrete time}

We now convert from continuous to discrete time

$$
H_{B}[k]=H_{B}\left(\mathrm{e}^{\mathrm{j} \omega}\right), \quad 0 \leq k \leq N-1, \quad \omega=\frac{2 \pi k}{N} .
$$

The linear-phase factor $\exp (-\mathrm{j} 2 \pi f \alpha)$ is then given by

$$
\exp \left\{-\mathrm{j} 2 \pi k \frac{M_{1}}{2} \frac{f_{s}}{N}\right\} \text {, }
$$

where $M_{1}$ is the length of the desired impulse response. The frequency response of the filter is

$$
\begin{gathered}
H_{B}[k]=\sqrt{F\{B[n]\}} \exp \left\{-\mathrm{j} 2 \pi k \frac{M_{1}}{2} \frac{f_{s}}{N}\right\}, \\
0 \leq k \leq N / 2,
\end{gathered}
$$

\footnotetext{
${ }^{1}$ The Wiener-Khinchin theorem states that the autocorrelation function of a wide-sense-stationary random process has a spectral decomposition given by the power spectrum of that process.
} 


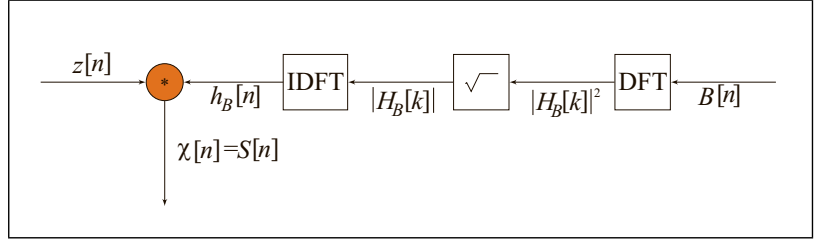

Figure 1. Block diagram of signal processing steps for generating scintillations. Gaussian white noise $z[n]$ is convolved with an impulse response $h_{B}[n]$ which is based on the covariance $B[n]$ of the turbulence spectrum.

where $F\{\}$ is the Discrete Fourier Transform (DFT). The desired impulse response is real-valued. Therefore we have because of Hermitian symmetry

$$
\begin{array}{ll}
H_{B}[k]=H_{B}\left(\mathrm{e}^{\mathrm{j} \omega}\right), & 0 \leq k \leq N / 2, \omega=\frac{2 \pi k}{N}, \\
H_{B}[N-k]=H_{B}^{*}[k], & 1 \leq k \leq N / 2-1 .
\end{array}
$$

In fact, because both $B[n]$ and $\left|H_{B}[k]\right|$ are real and even, one can use the type-1 Discrete Cosine Transform (DCT) instead of the DFT in equation (29). The impulse response of the filter is obtained by taking the Inverse Discrete Fourier Transform (IDFT)

$$
\begin{aligned}
h_{B}[n] & =F^{-1}\left\{H_{B}[k]\right\} \\
& =F^{-1}\left\{\sqrt{F\{B[n]\}} \exp \left\{-\mathrm{j} 2 \pi \frac{M_{1}}{2} k \frac{f_{s}}{N}\right\}\right\} .
\end{aligned}
$$

Scintillations are finally obtained through the convolution of the impulse response $h_{B}[n]$ with Gaussian white noise $z[n]$

$$
\chi[n]=S[n]=\left(z * h_{B}\right)[n] .
$$

The first $M_{1} / 2$ samples would have to be dropped because of the filter delay. A block diagram of the steps is shown in Figure 1.

\subsection{Apply scintillations to signal}

Now that we can generate sequences of fluctuations, we need to apply these to a carrier signal $x(t)$ or $x[n]$. According to equation (22) the log-amplitude fluctuations can be applied in a multiplicative manner, which is indeed the case of a signal with a sufficiently small bandwidth, like for example a monochromatic signal. However, the variance of the fluctuations is frequency-dependent, and since in practice broadband signals are commonly used, a method is sought for applying the fluctuations to a broadband signal.

One possible method would be to decompose the input signal in complex exponentials with the Inverse Discrete Fourier Transform, and apply per complex exponential the log-amplitude and phase fluctuations which, when combined, can be written as a complex exponential as shown in equations (10) and (11).

A computationally less demanding method is to instead filter the carrier signal with multiple band-pass filters, and proceed with applying fluctuations to each of the bandpass filtered signals and combining the resulting signals. Scintillations would be computed for the center frequencies of the bands. The amplitude fluctuations could be applied through multiplication and the phase fluctuations could be converted to time-delay fluctuations (see equation (35)) and applied with a Variable Delay Line (VDL).

\subsection{Scintillations as time-variant filter}

A more efficient method to take into account the frequency-dependence of the scintillations, would be to instead convolve the carrier signal with a time-variant filter that is updated fast enough to represent the fluctuations.

The fluctuations $\chi$ and $S$, at each time step computed for $M_{2}$ frequencies, can be merged into a complex phase (see equation (11))

$$
\psi[n]=\exp (\chi[n]+\mathrm{j} S[n])
$$

Adding a linear-phase factor as was done with $h_{B}[n]$ and taking the Inverse Discrete Fourier Transform will result in an impulse response for every instance in time $n$. Convolution of the carrier signal $x[n]$ with this time-variant filter will result in a log-amplitude and phase modulated signal. The first $M_{2} / 2$ samples will have to be dropped to correct for the filter delay.

Because the fluctuations are relatively low-frequent they can be sampled at a much lower sample frequency than the carrier signal. For a correlation length $L$ of 10 meters and a transverse speed $v_{\perp}$ of 2 meters per second the correlation time would be 5 seconds. If we would sample the sequence of fluctuations at 5 times the maximum bandwidth of the filter, which is proportional to the inverse of the correlation time $\tau_{0}$,

$$
f_{s}=5 / \tau_{0}
$$

the required sample frequency would be 1 Hertz.

In practice one might want to interpolate the impulse response as it changes over time, but because the phase also changes with time this can be problematic. Aside from converting to a minimum-phase representation we can also create a linear-phase filter for which the magnitude changes with time but the phase does not. If the phase fluctuations are linear-phase, then they can be applied to the carrier signal $x[n]$ with a Variable Delay Line. In the Gaussian model the phase fluctuations scale as $\sigma_{S}^{2} \sim f^{2}$. A scatterer affects all frequencies, and therefore the fluctuations of different frequencies move together, resulting in a linear-phase. We therefore write the phase fluctuation $\mathrm{d} S(t)$ as a propagation delay fluctuation

$$
\mathrm{d} t(t)=\mathrm{d} S(t) /(2 \pi f),
$$

or in discrete time

$$
\mathrm{d}[n]=\mathrm{d} S[n] /\left(2 \pi k f_{S}\right) .
$$

With the Gaussian turbulence spectrum it is straightforward to separate the covariance $B(\tau)$ into the correlation 
$C(\tau)$ and variance $\sigma$ as defined in respectively equations (16) and (14). That way we can compute a filter $h_{C}[n]$ that depends solely on the correlation $C(\tau)$, and scale this afterwards with the correct variances $\sigma_{\chi}$ and $\sigma_{S}$. The advantage is having to compute only one sequence of fluctuations, which is determined entirely by the covariance and $z[n]$, and can then be scaled with the (frequencydependent) variances $\sigma_{\chi}$ and $S$.

\subsection{Moving source}

Thus far it was assumed neither the source nor the receiver were moving, and that the fluctuations arise due to sampling a turbulent field that is moving by with constant speed at a fixed receiver position.

If the field is transported faster, the spatial fluctuations are sampled faster, and therefore the scintillations $\chi[n]=$ $S[n]$ are compressed in time corresponding to fluctuations with relatively higher frequencies.

In case the turbulent field is transported by wind and neither source nor receiver are moving, the source-receiver distance remains constant. If we instead consider a moving source of which the emitted sound samples the turbulent field, then the source-receiver distance $d$ generally changes and thus also the variances $\sigma_{\chi}$ and $\sigma_{S}$.

We will now consider a moving source. The transverse velocity is in this case the velocity component of the moving source, perpendicular to the wave propagation direction. This is the vector rejection of the source velocity vector $\mathbf{v}_{\mathbf{s}}$ from the unit vector $\hat{\mathbf{o}}$ that represents the orientation from source to receiver. The transverse speed is the norm of this vector

$$
v_{\perp}=\left\|\mathbf{v}_{\mathbf{s}}-\left(\mathbf{v}_{\mathbf{s}} \cdot \hat{\mathbf{o}}\right) \cdot \hat{\mathbf{o}}\right\| .
$$

The vector projection of the source velocity on the wave propagation direction is the component that is relevant for the Doppler shift. Therefore, as the Doppler shift is maximum the modulations are relatively low-frequent, whereas if the Doppler shift is zero, the modulations are relatively high-frequent.

When computing two sequences of scintillations, but for different transverse speeds, one does not obtain a compressed version of the other using the method as described thus far in this section, even when using the same seed for the Pseudo-Random Number Generator. This is because, by adjusting $v_{\perp}$, we effectively applied the compression on the filter $h_{C}[n]$ before convolution with $z[n]$. Therefore, the two resulting sequences will not look compressed in time, but instead just filtered differently. Even so, the generated sequence will have the desired statistical properties.

We can obtain the desired compression, i.e., scaling in time, if we instead perform the convolution between $z[n]$ and a time-invariant $h_{C}[n]$ computed for a fixed $\tau_{0}, \tau_{\text {ref }}$, and then resample the values of $\chi[n]$ and $S[n]$ at different times to take into account the change in correlation. Instead of having a constant sample timestep

$t_{k}=\frac{k}{f_{s}}=\sum_{i=0}^{k} \frac{1}{f_{s}}, \quad k=0,1, \ldots, \quad i=0,1, \ldots$

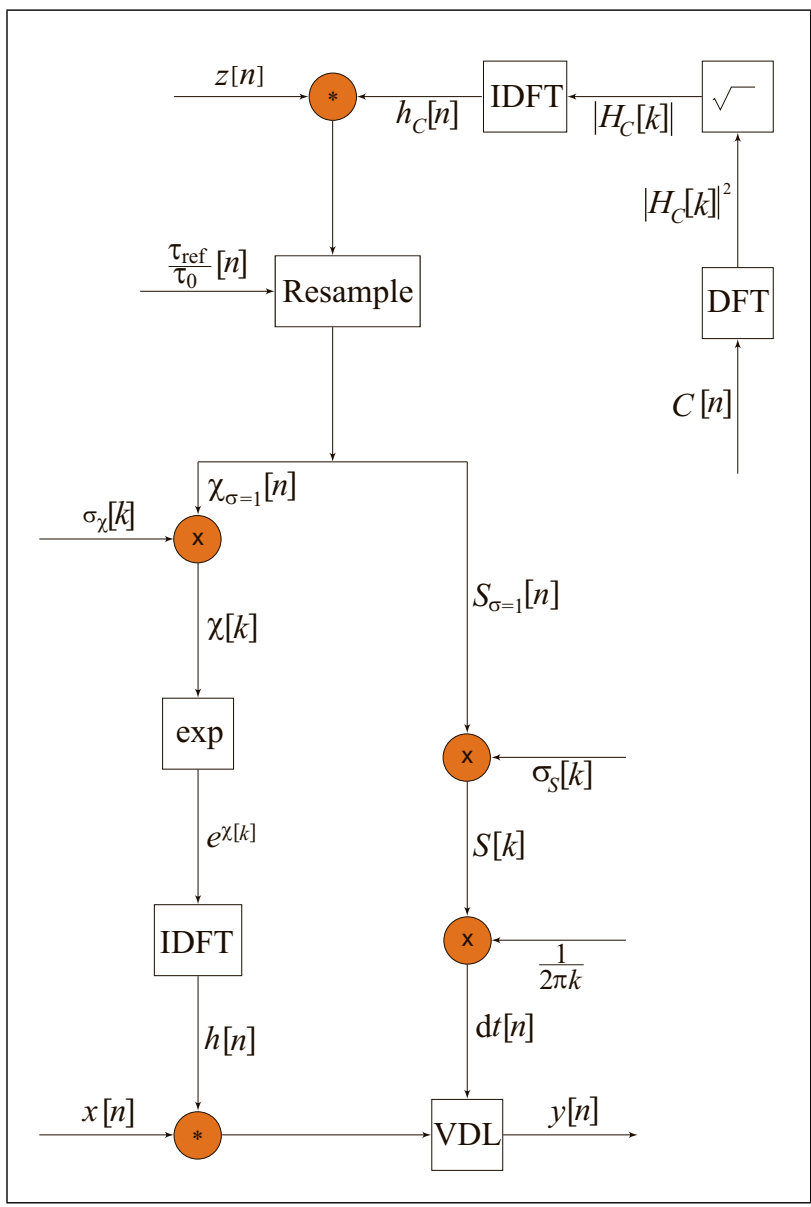

Figure 2. Block diagram of signal processing steps.

the sample time varies with correlation time

$t_{k}=\sum_{i=0}^{k} \frac{\tau_{\mathrm{ref}}}{\tau_{0, i}} \frac{1}{f_{s}}, \quad k=0,1, \ldots, \quad i=0,1, \ldots$

Figure 2 shows the full block diagram of the signal processing steps for generating scintillations and including them in an auralization.

\subsection{Saturation of the log-amplitude fluctuations}

According to equation (14) the fluctuations increase with distance and frequency indefinitely. For longer path lengths or stronger turbulence, the amplitude fluctuations gradually level off. Saturation of the amplitude fluctuations can be observed when measuring aircraft noise at distances of over a few kilometers. The standard deviation of the fluctuating sound pressure levels is in such cases limited to approximately $6 \mathrm{~dB}$ [15].

Saturation of the log-amplitude fluctuations can be included based on an analysis by Wenzel [17]. In Wenzel's approach the soundwave is split up in a coherent and incoherent wave. The amplitude of the coherent wave decreases over distance while the incoherent wave obtains the energy from the coherent wave. The coherent wave $p$ is written as

$$
\left\langle p p^{*}\right\rangle=\left(A_{m}^{2} / 4 \pi r^{2}\right) \exp \left(-2\left\langle\mu^{2}\right\rangle k^{2} r L\right)
$$




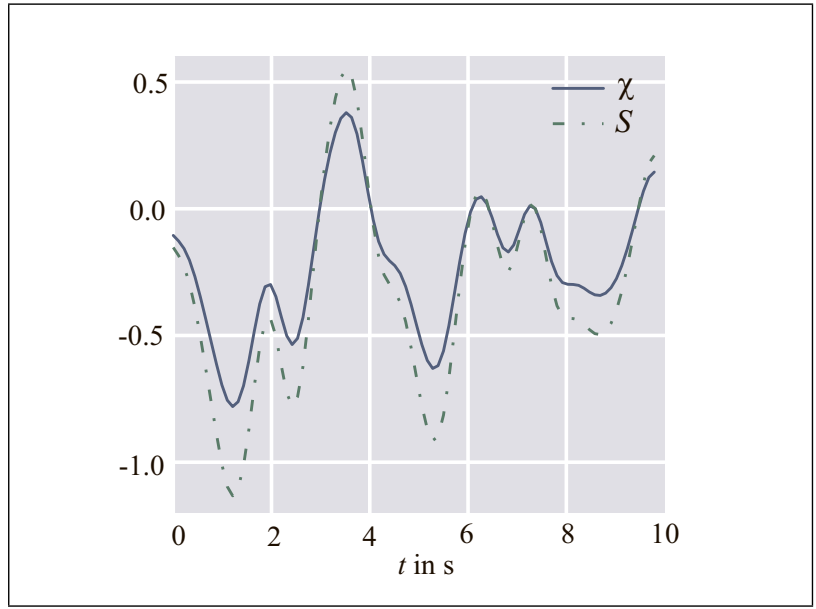

Figure 3. Amplitude and phase fluctuations as function of time. The fluctuations are less strong for the amplitude fluctuations due to saturation.

Wenzel defines the distance to saturation $r_{s}$ as the distance at which the coherent wave is reduced to $\mathrm{e}^{-1}$ of its original strength

$$
r_{s}=\frac{1}{2 \sigma_{\mu}^{2} k^{2} L}
$$

Saturation of the log-amplitude fluctuations can now be included by multiplying the log-amplitude with a correction factor. The variance of log-amplitude fluctuations that includes saturation $\sigma_{\chi}$, is given by

$$
\sigma_{\chi, s a t}=\sigma_{\chi} \frac{1}{1+r / r_{s}}
$$

\section{Results}

\subsection{A single tone}

To demonstrate the model we shall now consider a tone of $1000 \mathrm{~Hz}$ and apply modulations to the tone. The following parameters are used: $c=343.2 \mathrm{~m} / \mathrm{s}, \sigma_{\mu}^{2}=3 \cdot 10^{-6}, L=$ $1.1 \mathrm{~m}, v_{\perp}=2.0 \mathrm{~m} / \mathrm{s}$ and $d=500 \mathrm{~m}$. Saturation according to equation (42) is also included. The filter $h_{c}$ is designed to have 8192 taps. The correlation time is then $\tau=L / v_{\perp}=$ $0.55 \mathrm{~s}$ and the sample frequency at which the modulations are generated $5 / \tau=9.1 \mathrm{~Hz}$.

Figure 3 shows the time series of amplitude and phase fluctuations that were generated. Figure 4 shows the tone along with a modulated version of the tone. The tone was sampled at $8000 \mathrm{~Hz}$.

The log-amplitude and phase move in-phase. The value for the log-amplitude is slightly lower than that of the phase because of saturation. As expected the phase fluctuations cause spectral broadening of the tone as can be seen in Figure [18].

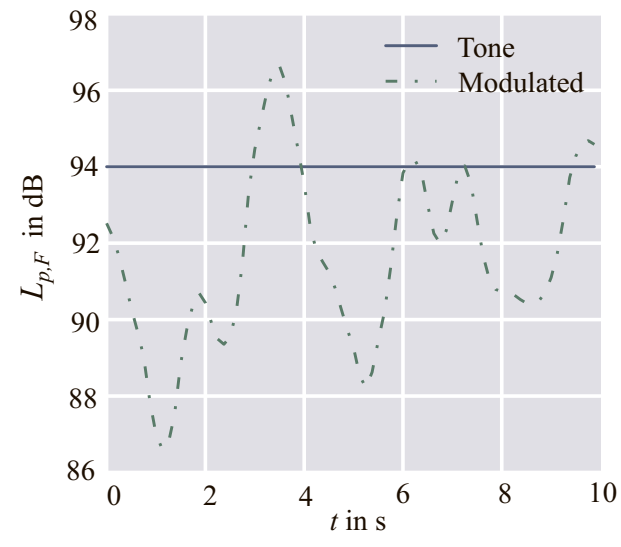

Figure 4. Sound pressure level of the tone as function of time, with and without fluctuations.

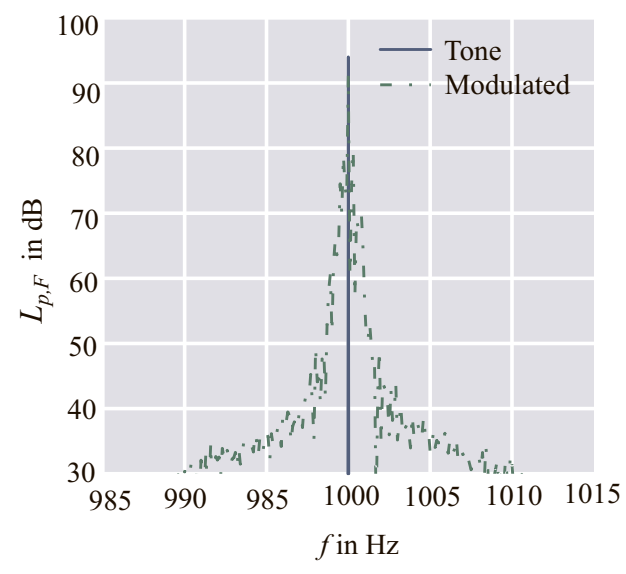

Figure 5 . The power spectrum of the tone shows the broadening due to the phase fluctuations.

\subsection{Scintillations as function of transverse speed}

By changing the transverse speed the refractive-index field is sampled at a different speed. This effectively changes the correlation length and shifts the frequency range that is covered by our applied filter [19].

Figure 6 shows the correlation function for different transverse speeds. With a high transverse speed the correlation drops faster, and this will result in relatively more high-frequency fluctuations, as is shown in Figure 7.

\subsection{Application in auralization of aircraft}

The method was developed in order to create more realistic sounding auralizations of aircraft. An aircraft moves at high speed through the atmosphere. A transverse speed is computed for each propagation path separately. Because the correlation time, distance and propagation varies between the two paths, decorrelation occurs. Figures 8 and 9 show spectrograms respectively with and without turbulence. The vertical lines that can be seen are the amplitude modulations. 


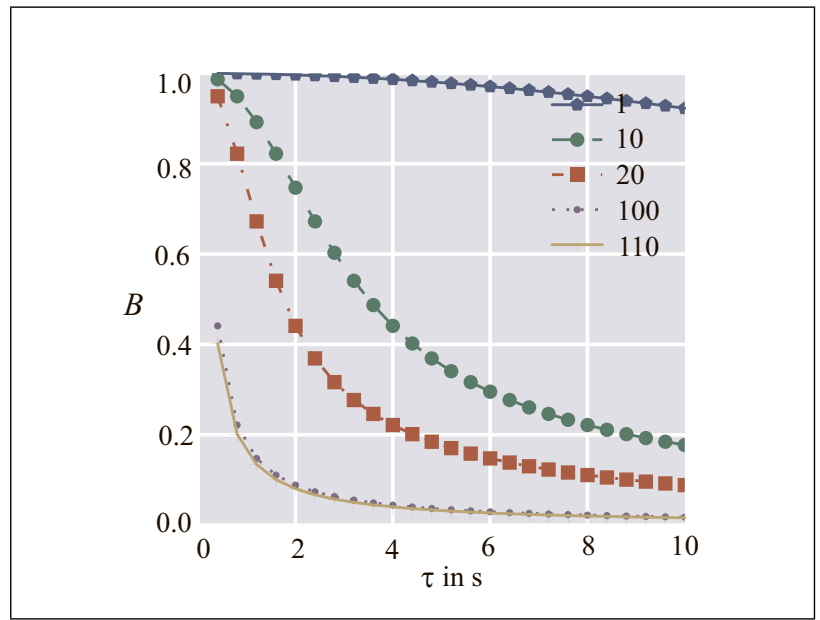

Figure 6. Correlation as function of time for different transverse speeds given in meters per second.

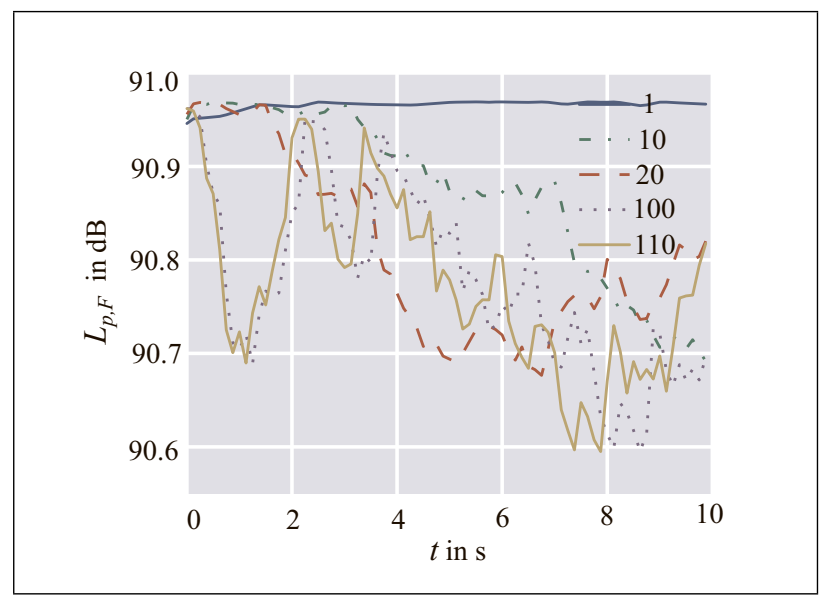

Figure 7. Sound pressure level as function of time for scintillations corresponding to different transverse speeds given in meters per second. The same $z[n]$ were used for each case. A compression in time of the modulations with increasing velocity can be observed.

Earlier it was assumed that the correlation length was much smaller than the Fresnel zone size. In this auralization the aircraft is moving close to the receiver. When the aircraft is closest, the distance is almost entirely given by the height which was approximately 100 meters. The correlation length was set at 20 meters. In that case the Fresnel zone is larger than the correlation length for the lower frequencies, and equation (14) is not valid. Instead, the log-amplitude and phase variances should scale with respectively $d^{3}$ and $2 k^{2} d$ instead of $k^{2} d$ [12].

\section{Conclusion}

Fluctuations in the refractive-index field due to variations in temperature and wind affects sound propagation and causes audible modulations. A method was presented for generating sequences of modulations and applying these to monochromatic as well as broadband signals.

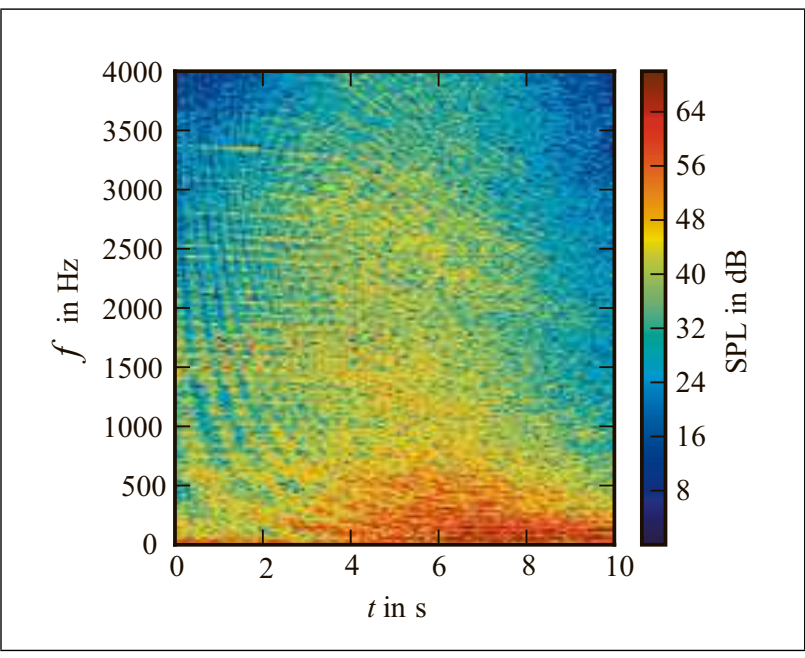

Figure 8. Spectrogram of an auralization of an aircraft taking off. Scintillations were not included. Visible are the ground effect and the Doppler shift. In the initial seconds a high amount of tonal components are visible.

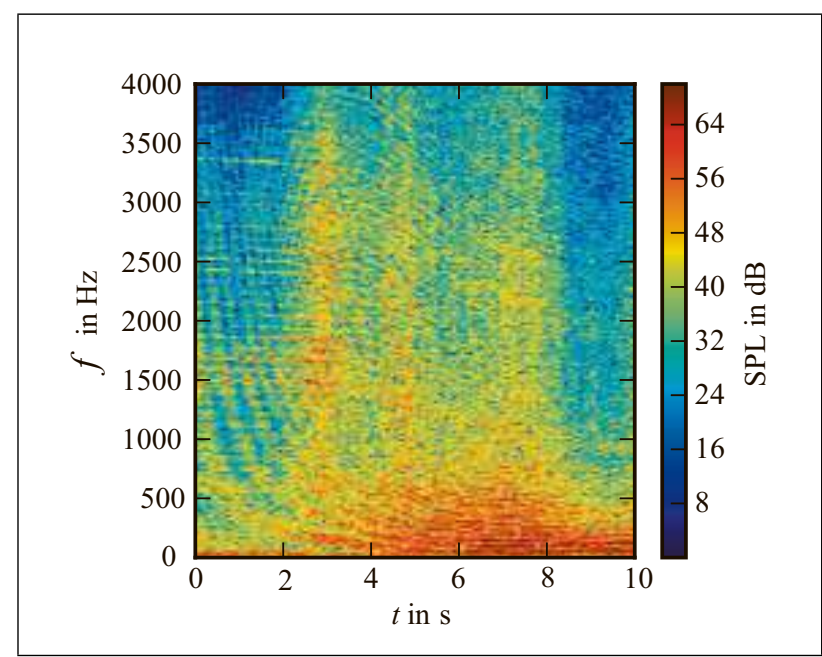

Figure 9. Spectrogram of the same event as in Figure 8, however, this time with scintillations included. Because of the high speed at which the aircraft samples the refractive-index field the scintillations are relatively high-frequent, resulting in vertical lines.

A Rytov approximation to first-order refractive-index flucutuations results in a complex phase which we can write as a log-amplitude $\chi$ and phase $S$ fluctuation. The propagating sound is modelled as a time-varying channel where we consider two sequences, one for the logamplitude fluctuations, and another for the phase fluctuations.

The fluctuations are frequency-dependent and therefore a filter was designed to take that into account. A Gaussian turbulence spectrum was considered, but the general method can be used with other turbulence spectra as well. The Von Karman spectrum describes real turbulence spectra typically better than the Gaussian spectrum, however, the Von Karman spectrum is computationally much more demanding. 
Examples are shown where the method is applied to a tone and to an aircraft auralization. The aircraft auralization spectrogram shows several spikes corresponding to amplitude modulations as well as an increase in the amount of decorrelation. Furthermore the transverse velocity dependence on the frequency content of the modulations is demonstrated.

According to the authors the method results in more realistic sounding auralizations, but this has not been validated yet with listening tests. The implementation of the model that was used in this paper to generate the figures can be found at [20].

\section{Acknowledgement}

The research leading to these results has received funding from the People Programme (Marie Curie Actions) of the European Union's Seventh Framework Programme FP7/2007-2013 under REA grant agreement number 290 110, SONORUS “Urban Sound Planner".

\section{References}

[1] K. Heutschi, R. Pieren, M. Müller, M. Manyoky, U. W. Hayek, K. Eggenschwiler: Auralization of Wind Turbine Noise: Propagation Filtering and Vegetation Noise Synthesis. Acta Acustica united with Acustica 100 (2014) 13-24.

[2] J. Scheuren, W. Kropp, J. Forssén: SONORUS - Training Urban Sound Planners by Education and Research. Proceedings of Forum Acusticum, 7th Forum Acusticum (2014).

[3] F. Rietdijk, K. Heutschi, C. Zellmann, M. June: Determining an empirical emission model for the auralization of jet aircraft. Proceedings of EuroNoise 2015, Maastricht, The Netherlands, 2015, 781-784.

[4] H.-C. Shin, C. Hall, D. Crichton: Auralisation of turbofan engine noise components. Collection of Technical Papers 12th AIAA/CEAS Aeroacoustics Conference, 2006, 28412854.

[5] M. Arntzen, D. G. Simons: Ground reflection with turbulence induced coherence loss in flyover auralization. International Journal of Aeroacoustics 13 (2014) 449-462.

[6] M. Arntzen: Aircraft noise calculation and synthesis in a non-standard atmosphere. TU Delft, Delft University of Technology, dec 2014.
[7] A. Minard, S. Hourcade, C. Lambourg, P. Boussard: Sound synthesis and 3D sound rendering of aircraft flyovers with controllable parameters. 22nd AIAA/CEAS Aeroacoustics Conference, Lyon, 2016.

[8] A. Jurado-Navas, J. M. Garrido-Balsells, M. Castillo-Vázquez, A. Puerta-Notario: Numerical Simulation of Physical and Engineering Processes. 2011, Kap. 7, 612.

[9] J. Forssén: Calculation of Noise Barrier Performance in a Turbulent Atmosphere by Using Substitute Sources With Random Amplitudes. Proc. 9th Long Range Acoustic Propagation Symposium, The Hague, The Netherlands, 2000, 16-26.

[10] F. Rietdijk, K. Heutschi, J. Forssén: Modelling sound propagation in the presence of atmospheric turbulence for the auralisation of aircraft noise. Forum Acusticum 2014, Krakow, 2014.

[11] F. Rietdijk, K. Heutschi, J. Forssén: Modelling sound propagation in the presence of atmospheric turbulence for the auralization of aircraft noise. The Journal of the Acoustical Society of America 136 (oct 2014) 2286-2286.

[12] A. Ishimaru: Wave Propagation and Scattering in Random Media. Wiley-IEEE Press, New York, 1997.

[13] V. Tatarskii: The effects of the turbulent atmosphere on wave propagation. ISBN 070650680 4, 1971, 472.

[14] E. Salomons: Computational Atmospheric Acoustics. ISBN 1402003900, 2001.

[15] G. A. Daigle, J. E. Piercy, T. F. W. Embleton: Line-ofsight propagation through atmospheric turbulence near the ground. Journal of the Acoustical Society of America $\mathbf{7 4}$ (1983) $1505-1513$.

[16] V. E. Ostashev, D. K. Wilson: Acoustics in moving inhomogeneous media. ISBN 9780415564168, second ed. 2015, 541.

[17] A. R. Wenzel: Saturation effects associated with sound propagation in a turbulent medium. American Institute of Aeronautics and Astronautics (1975).

[18] V. E. Ostashev, D. K. Wilson, A. Finn, K. Rogers, E. Barlas: Spectral broadening of acoustic tones generated by unmanned aerial vehicles in a turbulent atmosphere. Journal of the Acoustical Society of America 140 (oct 2016) 3119 3119 .

[19] D. K. Wilson, J. G. Brasseur, K. E. Gilbert: Acoustic scattering and the spectrum of atmospheric turbulence. The Journal of the Acoustical Society of America 105 (1999) 30-34.

[20] F. Rietdijk: Scintillations. DOI: 10.5281/zenodo.202387, URL: https://doi.org/10.5281/zenodo.202387, 2016. 